cross comparison of hundreds of cases did common patterns emerge, culminating in Osler's landmark text, The Principles and Practice of Medicine.

Haffner and colleagues should be commended for reporting such a fascinating anecdote (1), but the effect of their work will only be realized if we can collect and assemble this type of data across hundreds of cases (Figure 1). The growing penetration of genomic sequencing into clinical medicine has led to increased calls for the creation of various types of medical "data commons" that will allow comparisons between individual cases (6). Perhaps we are entering an era in which " $N$ of 1 " cases are here to stay.

\section{Acknowledgments}

Support was provided by the National Cancer Institute, NIH, under award numbers 5T32CA160001, R01-CA155169, P50-CA092629, and U01 CA141502. The content is solely the responsibility of the authors and does not necessarily represent the official views of the NIH.

Address correspondence to: Charles L. Sawyers, Memorial Sloan-Kettering Cancer Center, 1275 York Avenue, New York, New York 10021, USA. Phone: 646.888.2594; Fax: 646.888.2595; E-mail: sawyersc@ mskcc.org.

1. Haffner MC, et al. Tracking the clonal origin of lethal prostate cancer. J Clin Invest. 2013
123(11):4918-4922.

2. Yachida S, et al. Distant metastasis occurs late during the genetic evolution of pancreatic cancer. Nature. 2010;467(7319):1114-1117.

3. Gerlinger $M$, et al. Intratumor heterogeneity and branched evolution revealed by multiregion sequencing. NEngl J Med. 2012;366(10):883-892.

4. Iyer $\mathrm{G}$, et al. Genome sequencing identifies a basis for everolimus sensitivity. Science. 2012; 338(6104):221

5. Eastman P. Online first: New FDA 'breakthrough' designation likely to speed cancer drug approvals. Oncology Times Web site. http://journals.lww. com/oncology-times/blog/onlinefirst/pages/post. aspx?PostID=578. Updated November 17, 2012. Accessed September 25, 2013.

6. National Research Council (US) Committee on A Framework for Developing a New Taxonomy of Disease. Toward precision medicine: Building a knowledge network for biomedical research and a new taxonomy of disease. Washington DC, USA: The National Academies Press; 2011.

\title{
Antifibrotic vitamin D analogs
}

Joseph V. Bonventre

Renal Division, Department of Medicine, Brigham and Women's Hospital, Boston, Massachusetts, USA

\begin{abstract}
Chronic kidney disease is associated with progressive kidney fibrosis, which disrupts normal kidney function. There is a great need for treatments to reduce renal fibrosis. In this issue of the JCI, Ito and colleagues report the development of synthetic ligands of the vitamin $D$ receptor that target the TGF- $\beta$-SMAD signaling pathway, which is known to regulate fibrosis-associated gene expression, without inducing VDR-associated genes. These ligands ameliorated renal fibrosis in two different mouse models. This study justifies further investigation of these and related compounds for treatment of humans with chronic kidney disease or other diseases characterized by fibrosis.
\end{abstract}

\section{TGF- $\beta$ signaling promotes chronic kidney disease}

Chronic kidney disease (CKD) affects approximately $10 \%$ of the adult population in the developed world (1). A dominant feature of most forms of CKD is the development of kidney fibrosis, which results in progressive loss of kidney function, enhanced susceptibility to cardiovascular disease, and potentially end stage

Conflict of interest: The author or his family hold equity interests in Patientkeeper, AMAG, Pacific Biosciences, MediBeacon, Theravance, Sentien, DxNow, and DRP. Joseph V. Bonventre is a consultant for Janssen RND, Keryx, and editor of Seminars In Nephrology. Joseph V. Bonventre holds patents on KIM-1, which have been assigned to Partners Healthcare which has licensed them to Sekisui, Novartis, Johnson and Johnson, BiogenIdec, and a number of research reagent providers. He has received research support from NovoNordisk.

Citation for this article: J Clin Invest. 2013; 123(11):4570-4573. doi:10.1172/JCI72748. renal disease. CKD progression occurs even if the original cause of the kidney disease is no longer operant. Despite the worldwide prevalence of CKD, few therapeutic strategies have any impact on the prevention or treatment of fibrotic kidney disease.

One factor with a prominent role in fibrosis development in the kidney and other organs is TGF- $\beta$, which signals through the TGF- $\beta$-SMAD signaling pathway (2). TGF- $\beta$ binds to cell surface type I and II serine/threonine receptor kinases, resulting in phosphorylation of SMAD2 and SMAD3, which are then released into the cytosol and bind in a complex with SMAD4. After translocation to the nucleus, the SMAD2/3/4 complex localizes to SMAD-binding elements within the genome to modulate expression of profibrotic and other target genes (Figure 1 and ref. 3). During kidney injury, a major source of TGF- $\beta$ is proximal tubule epithelial cells, some of which are arrested in cell cycle phase $\mathrm{G}_{2} / \mathrm{M}$ (4). The release of TGF- $\beta$ and other factors by the damaged epithelial cells and infiltrating inflammatory cells act in a paracrine fashion to activate interstitial fibroblasts/pericytes. Once activated, these interstitial cells convert to proliferative myofibroblasts and maladaptively deposit extracellular matrix, which leads to interstitial fibrosis $(2,5)$. Since TGF- $\beta$ plays a major role in this pathophysiological response, there have been attempts to therapeutically interrupt the TGF- $\beta$ signaling pathway with either small molecules or antibodies. Some of these approaches are currently in phase I and II clinical trials (6); however, there is still no accepted TGF- $\beta$ targeted therapy for kidney fibrosis.

\section{Vitamin D signaling in the kidney}

Interaction of 1,25-dihydroxyvitamin $\mathrm{D}_{3}$ $\left[1,25(\mathrm{OH})_{2} \mathrm{D}_{3}\right]$ with the vitamin $\mathrm{D}$ receptor (VDR) modulates the transcription of more than 200 genes (7). The VDR forms a heterodimer with retinoid $X$ receptors (RXRs) that together promote the recruitment of nuclear coactivators and the lysine acetyltransferase CBP/p300, which provides access for the basal transcriptional machinery through histone acetylation (Figure 1 and ref. 8). The VDR is expressed in more than 30 different tissues (9), including the kidney. VDR- 


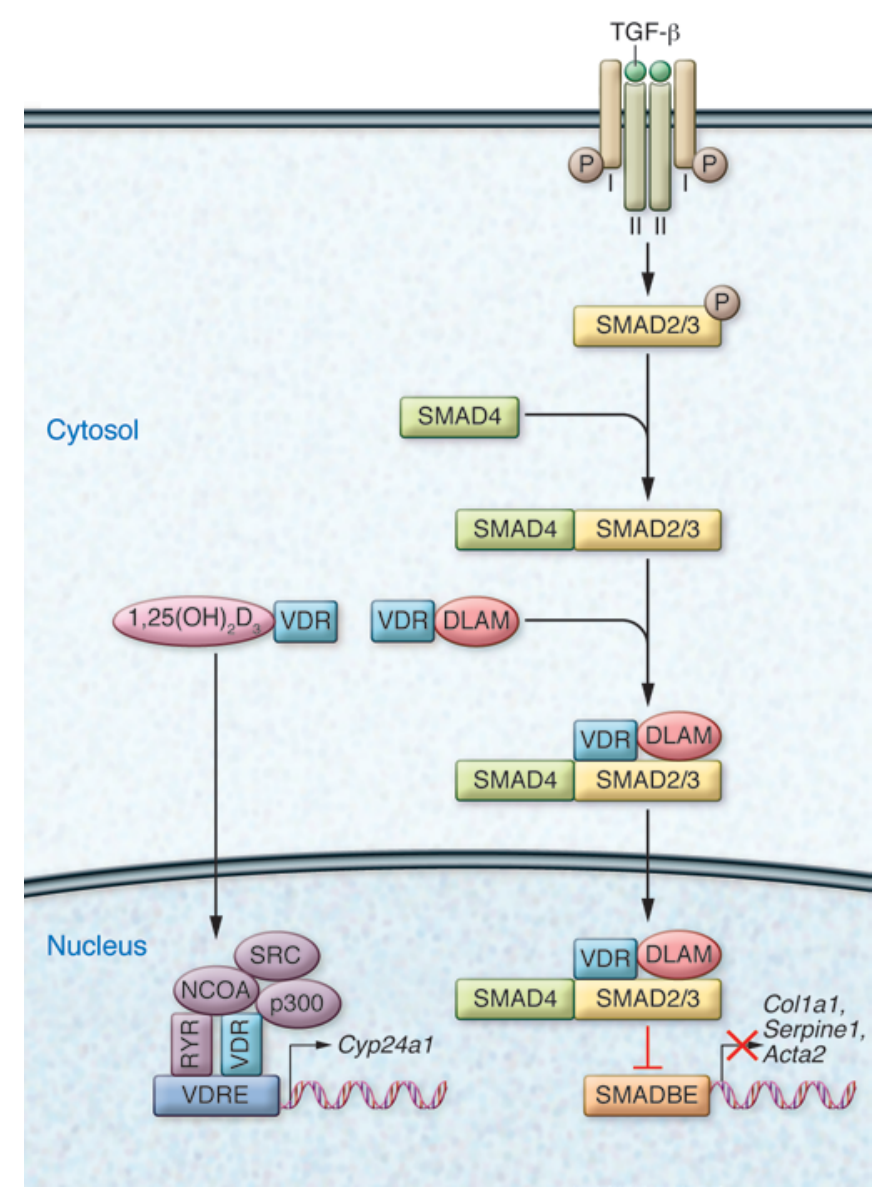

\section{Figure 1}

DLAMs interact with the VDR and block TGF- $\beta$ signaling by preventing binding to the SMAD3-binding element (SMADBE). This prevention of binding prevents expression of profibrotic genes. The DLAMs do not affect classical $1,25(\mathrm{OH})_{2} \mathrm{D}_{3}$-VDR-potentiated actions on genes, such as Cyp24a1, due to interaction with VDRE. Cyp24a1 encodes $1,25(\mathrm{OH})_{2} \mathrm{D}_{3}-24$-hydroxylase, a mitochondrial enzyme that degrades $1,25(\mathrm{OH})_{2} \mathrm{D}_{3}$ and hence plays an important role in calcium homeostasis. Normally, TGF- $\beta$ signals by binding to cell surface type I and type II serine/threonine receptors, which release phosphorylated SMAD3 into the cytosol, where it interacts with SMAD4, translocates to the nucleus, binds to the SMADBE in genes, and upregulates profibrotic gene expression. NCOA, nuclear coactivators; SRC, steroid receptor coactivator.
RXR heterodimers interact with VDR elements (VDREs) within the genome, which are often in promoter regions of genes. Many $1,25(\mathrm{OH})_{2} \mathrm{D}_{3}$-regulated genes have pleiotropic roles, including in intestinal calcium/phosphate reabsorption and bone remodeling, which are both important aspects of calcium homeostasis.

A number of epidemiological (10) and interventional (11) studies suggest that vitamin $\mathrm{D}$ may mitigate albuminuria or prevent progression of kidney dysfunction. Additionally, many laboratory studies have identified a large number of vitamin Dregulated cellular and tissue processes that could potentially suppress renal fibrosis and benefit kidney structure and function.

\section{Targeting VDR to prevent TGF- $\beta$ - SMAD-induced kidney fibrosis}

In this issue of the JCI, Ito et al. evaluated inhibition of VDR signaling as a therapeutic strategy for suppressing renal fibrosis development (12). They found that $1,25(\mathrm{OH})_{2} \mathrm{D}_{3}$-bound VDR inhibits the TGF- $\beta$-SMAD signaling pathway directly through interaction with SMAD3.
In a mouse model of unilateral ureteral obstruction (UUO), which is well known for its rapid development of fibrotic kidney disease, treatment with $1,25(\mathrm{OH})_{2} \mathrm{D}_{3}$ markedly reduced TGF- $\beta$-stimulated profibrotic gene expression and fibrosis development. Fibrosis was inhibited despite the observation that this treatment did not prevent the UUO-induced increase in TGF- $\beta$ and subsequent increase in SMAD2 and SMAD3 phosphorylation. In murine renal epithelial cells stimulated with TGF- $\beta$, $1,25(\mathrm{OH})_{2} \mathrm{D}_{3}$ treatment did not affect SMAD2 or SMAD3 phosphorylation or their translocation to the nucleus, but did mitigate the upregulation of a number of TGF- $\beta$ target genes implicated in fibrosis. ChiP assays revealed that the TGF- $\beta$-enhanced interaction between SMAD3 and the Serpine1 (encoding plasminogen activator inhibitor-1 [PAI1]) or Acta 2 (encoding $\alpha$-SMA) promoters was markedly suppressed in the presence of $1,25(\mathrm{OH})_{2} \mathrm{D}_{3}$. Binding of SMAD3 to the SMAD3 DNA-binding element was blocked by the presence of the VDR-ligand binding domain and $1,25(\mathrm{OH})_{2} \mathrm{D}_{3}$.
Ito and colleagues generated two synthetic analogs of $1,25(\mathrm{OH})_{2} \mathrm{D}_{3}$ that interact with the VDR and inhibit TGF- $\beta$-induced SMAD signaling without activating classical VDR-mediated genes, thus preventing the development of hypercalcemia. The crystal structure of the VDR bound to $1,25(\mathrm{OH})_{2} \mathrm{D}_{3}$ was previously solved and revealed that $1,25(\mathrm{OH})_{2} \mathrm{D}_{3}$ associates with helix 12 (H12) to stabilize VDR, which facilitates interactions with coactivators (13). Removal of H12 not only prevented VDR-dependent transcriptional activity, but also abolished inhibition of TGF- $\beta$ dependent transcription; therefore, this region of H12 is important for interaction with SMAD3. Since the physiological $1,25(\mathrm{OH})_{2} \mathrm{D}_{3}$ analog, $1 \alpha, 25$-dihydroxyvitamin $\mathrm{D}_{3}-26,23$-lactone (1,25-lactone), has a $\gamma$-butyrolactone ring, the authors proposed that it could affect interaction with the $\mathrm{H} 12$ region differently than $1,25(\mathrm{OH})_{2} \mathrm{D}_{3}$. 1,25-lactone had no effect on TGF- $\beta$-induced SMAD3 phosphorylation, but blocked TGF- $\beta$ signaling; however, this molecule did have some agonistic effect on VDR-mediated transcription of Cyp24a1 
[encoding 1,25(OH) ${ }_{2} \mathrm{D}_{3}$-24-hydroxylase]. The authors found that 1,25-lactone in complex with the ligand-binding domain of VDR associates differently with residues His305 and His 397 than does $1,25(\mathrm{OH})_{2} \mathrm{D}_{3}$. Based on the structure of 1,25-lactone and its effect on VDR activity, Ito et al. designed 1 $\alpha, 25$-dihydroxyvitamin $\mathrm{D}_{3}$-26,23-lactam (DLAM) derivatives, which are analogs of 1,25-lactone that mimic VDR ligands (14). DLAM candidates were chosen for their ability to alter the configuration of the $\mathrm{H} 12$ region.

In the UUO model, the authors found that administration of DLAMs reduced the level of SMAD3-dependant mRNAs, but did not increase Cyp24a1 mRNA levels (12). DLAM treatment reduced mRNA levels of Serpine1, Acta2, and Col1a1 (encoding type I collagen) in the obstructed kidney, and ChIP analysis revealed suppressed binding of SMAD3 to the Serpine1 promoter after DLAM treatment. Thus, TGF- $\beta-S M A D$ signaling was inhibited by DLAM treatment, but other actions of the VDR were not stimulated (Figure 1). The authors also found that serum calcium levels were unaffected by DLAM treatment.

Evaluation of paracalcitol, a VDR agonist used to treat secondary hyperparathyroidism in CKD and known to produce less hypercalcemia than $1,25(\mathrm{OH})_{2} \mathrm{D}_{3}$, revealed that unlike with DLAM treatment, Cyp24a1 expression was increased. While paracalcitol decreased mRNA levels of Col1a1 and Acta2, it did not decrease the TGF- $\beta$ target gene Serpine1, in obstructed kidneys. Furthermore, in contrast to DLAM, paracalcitol increased serum calcium levels considerably. DLAM treatment in a second mouse model of renal fibrosis, folic acid nephropathy, had similar effects to those found in the UUO model. These included decreased levels of Serpine1, Acta2, and Col1a1 mRNA and no change in VDR-dependent mRNAs. Additionally, progressive fibrosis and renal failure were inhibited in DLAM-treated mice.

A recent important related study demonstrated that treatment with a synthetic VDR agonist, calcipotriol, is protective against liver fibrosis and does not cause hypercalcemia (15). Ding et al. found that TGF- $\beta$ causes redistribution of genomewide VDR-binding sites in hepatic stellate cells, which are comparable to interstitial fibroblasts/pericytes in the kidney. In the presence of calcipotriol, SMAD3 occupancy on profibotic genes is reduced; therefore, carbon tetrachloride-treated mice exhibit less liver fibrosis in the presence of calcipotriol.

\section{Implications and future directions}

The study by Ito et al. (12) provides intriguing promise for kidney fibrosis treatment and prevention. The authors made informative distinctions between TGF- $\beta$-dependent effects on profibrotic gene expression and classical $1,25(\mathrm{OH})_{2} \mathrm{D}_{3}$ VDR-dependent transcription to motivate development of antifibrotic agents that do not cause hypercalcemia in mice. In particular, they applied sophisticated knowledge of the VDR structure and its interaction with $1,25(\mathrm{OH})_{2} \mathrm{D}_{3}$ and a naturally occurring analog, 1,25-lactone (16), to derive compounds with anti-TGF- $\beta$ activity that do not promote the expression of VDRdependent genes. It is possible that these or related agents may serve to reduce fibrosis in humans.

A word of caution is indicated, however. Protective effects of $1,25(\mathrm{OH})_{2} \mathrm{D}_{3}$ in humans have been reported in large epidemiological studies (17) and may relate to VDR-mediated responses that are unrelated to TGF- $\beta$ mitigation. $1,25(\mathrm{OH})_{2} \mathrm{D}_{3}$ has also been reported to have important effects on innate and adaptive immunity as well as protective effects on the cardiovascular system, cancer susceptibility, and neurocognitive function (18). Therefore, an agent specifically targeting the TGF- $\beta$ pathway may prove ineffective if other VDR-mediated effects of $1,25(\mathrm{OH})_{2} \mathrm{D}_{3}$ are more important or are necessary to complement the anti-TGF- $\beta$ effects of treatment. Additionally, it is unknown whether other noncalcemic gene targets of $1,25(\mathrm{OH})_{2} \mathrm{D}_{3}$ are stimulated by DLAMs, as the specificity of the DLAMs used to block TGF- $\beta$ signaling have not been evaluated beyond the lack of effects on Cyp24a1 transcription and the calcemic effect in animals.

An interesting aspect of this work is the characterization of the action of the $1,25(\mathrm{OH})_{2} \mathrm{D}_{3}$ physiological analog 1,25lactone. It is possible that 1,25-lactone represents an endogenous fibrosis inhibitor in organs, including the kidney, heart, lung, and liver. Could subjects with CKD have reduced levels of 1,25-lactone, which would exacerbate the detrimental profibrotic effects attributed to low levels of $1,25(\mathrm{OH})_{2} \mathrm{D}_{3}$ ? Are 1,25-lactone levels lower in African-Americans, who, as a group, have high incidence of CKD and albuminuria and lower levels of circulating vitamin D [25(OH)D] (19)? This study provides an elegant approach for systematically developing therapeutic targets for disease treatment. Is it possible that this general approach to developing targeted ligands will be effective in identifying specific beneficial analogs for other steroid hormones that are important therapeutically. Analogs of glucocorticoids that preserve beneficial effects while minimizing detrimental effects would be of great benefit in a number of diseases, including diseases of the kidney.

\section{Acknowledgments}

J.V. Bonventre is supported by NIH awards DK 39773 and DK 72381.

Address correspondence to: Joseph V. Bonventre, Renal Division, Brigham and Women's Hospital, Harvard Medical School, Harvard Institutes of Medicine, Rm 576, 4 Blackfan Circle, Boston, Massachusetts 02115, USA. Phone: 617.525.5960; Fax: 617.525.5965; E-mail: joseph_bonventre@ hms.harvard.edu.

1. Eckardt KU, et al. Evolving importance of kidney disease: from subspecialty to global health burden. Lancet. 2013;382(9887):158-169.

2. Kaissling B, Lehir M, Kriz W. Renal epithelial injury and fibrosis. Biochim Biophys Acta. 2013; 1832(7):931-939.

3. Massagué J. TGF $\beta$ signalling in context. Nat Rev Mol Cell Biol. 2012;13(10):616-630.

4. Yang L, Besschetnova TY, Brooks CR, Shah JV, Bonventre JV. Epithelial cell cycle arrest in G2/M mediates kidney fibrosis after injury. Nat Med. 2010; 16(5):535-543.

5. Bonventre JV, Yang L. Cellular pathophysiology of ischemic acute kidney injury. J Clin Invest. 2011; 121(11):4210-4221.

6. Akhurst RJ, Hata A. Targeting the TGF $\beta$ signalling pathway in disease. Nat Rev Drug Discov. 2012; 11(10):790-811.

7. Carlberg C, Seuter S. A genomic perspective on vitamin D signaling. Anticancer Res. 2009; 29(9):3485-3493.

8. Sutton AL, MacDonald PN. Vitamin D: more than a "bone-a-fide" hormone. Mol Endocrinol. 2003; 17(5):777-791.

9. Bookout AL, Jeong Y, Downes M, Yu RT, Evans RM, Mangelsdorf DJ. Anatomical profiling of nuclear receptor expression reveals a hierarchical transcriptional network. Cell. 2006;126(4):789-799.

10. de Boer IH, Ioannou GN, Kestenbaum B, Brunzell JD, Weiss NS. 25-Hydroxyvitamin D levels and albuminuria in the Third National Health and Nutrition Examination Survey (NHANES III). Am J Kidney Dis. 2007;50(1):69-77.

11. de Zeeuw D, et al. Selective vitamin D receptor activation with paricalcitol for reduction of albuminuria in patients with type 2 diabetes (VITAL study): a randomised controlled trial. Lancet. 2010;376(9752):1543-1551.

12. Ito I, et al. A nonclassical vitamin D receptor pathway suppresses renal fibrosis. J Clin Invest. 2013; 123(11):4579-4594

13. Rochel N, Wurtz JM, Mitschler A, Klaholz B, Moras D. The crystal structure of the nuclear receptor for vitamin D bound to its natural ligand. Mol Cell. 2000;5(1):173-179.

14. Nakano Y, et al. Practical synthesis and evaluation 
of the biological activities of $1 \alpha, 25$-dihydroxyvitamin D3 antagonists, 1alpha,25-dihydroxyvitamin D3-26,23-lactams. Designed on the basis of the helix 12-folding inhibition hypothesis. J Med Chem. 2006;49(8):2398-2406

15. Ding $\mathrm{N}$, et al. A vitamin $\mathrm{D}$ receptor/SMAD genomic circuit gates hepatic fibrotic response. Cell. 2013;153(3):601-613.

16. Ishizuka S, Ohba T, Norman AW. $1 \alpha, 25(\mathrm{OH})_{2} \mathrm{D}_{3}$
26,23-Lactone is a major metabolite of $1 \alpha, 25(\mathrm{OH})_{2} \mathrm{D}_{3}$ under physiological conditions. In: Norman AW, Schaefer K, Grigoleit HG, Herrath DV, eds. Vitamin D: Molecular, Cellular, And Clinical Endocrinology. Berlin, Germany: Walter de Gruyter; 1988:143-144.

17. Li YC. Vitamin D receptor signaling in renal and cardiovascular research. Semin Nephrol. 2013; 33(5):433-447.
18. Pludowski P, et al. Vitamin D effects on musculoskeletal health, immunity, autoimmunity, cardiovascular disease, cancer, fertility, pregnancy, dementia and mortality-a review of recent evidence. Autoimmun Rev. 2013;12(10):976-989.

19. Essien E, Goel N, Melamed ML. Role of vitamin $D$ receptor activation in racial disparities in kidney disease outcomes. Semin Nephrol. 2013; 33(5):416-424.

\title{
Mitochondrial ROS deficiency and diabetic complications: AMP[K]-lifying the adaptation to hyperglycemia
}

\author{
Dwight A. Towler
}

Sanford-Burnham Medical Research Institute and Florida Hospital Translational Research Institute, Orlando, Florida, USA. Department of Medicine, Endocrine Division, University of Florida, Gainesville, Florida, USA.

\begin{abstract}
Global, sustained production of ROS has deleterious effects on tissue structure and function and gives rise to biochemical and physiological changes associated with organ senescence. Specific, localized ROS metabolites generated by mitochondria and NADPH oxidases also transduce homeostatic information in response to metabolic, mechanical, and inflammatory cues. In this issue of the JCI, Dugan and colleagues demonstrate that mitochondrial-derived ROS, which is maintained by a feed-forward AMP kinase activation cascade, is reduced in diabetes and plays an adaptive role in preserving renal glomerular function during hyperglycemia. This enlightened view of mitochondrial ROS biology forces us to reconsider therapeutic approaches to metabolic disease complications such as diabetic nephropathy.
\end{abstract}

\section{Diabetes management: a complicated issue}

With an estimated 366 million individuals afflicted worldwide, the importance of diabetes' effects on human health and healthcare cannot be overstated (1). In the United States alone, the direct and indirect costs are staggering. Every year in the US, 250 billion dollars, approximately $1.5 \%$ of the country's entire gross domestic product, is spent on diabetes and its complications (2). The intrinsic cellular responses and vascular injury that arise from hyperglycemia result in a variety of costly complications such as neuropathies, retinopathies, and cardiovascular disease, which includes heart failure, stroke, myocardial infarction, and arteriosclerosis. Improvements in glycemic control alone are insufficient to fully mitigate diabetes-associated complications; therefore, clinical differences

Conflict of interest: The author has declared that no conflict of interest exists.

Citation for this article: J Clin Invest. 2013; 123(11):4573-4576. doi:10.1172/JCI72326. in the pathobiology of diabetic end organ complications should drive the search for adjunctive therapeutic approaches above and beyond glycemic control. For example, intensive control of both glucose and lipid intake has emerged as an effective strategy for reducing retinopathy (3), but other diabetes-associated complications such as nephropathy (4) and macrovascular disease (5) remain refractory to this focused metabolic strategy. Moreover, interactions between the metabolites produced in association with diabetes and chronic kidney disease synergize and have devastating effects on cardiovascular health (6). Innovative therapeutic approaches are necessary for diabetes treatment, and the pathways involved in mitochondrial oxidative stress have become attractive targets (7).

\section{Oxidative stress in diabetes}

It is widely appreciated that extracellular oxidative stress globally increases in both type 1 diabetes (T1D) and type 2 diabetes (T2D) $(8,9)$. Current opinion holds that diabetes-associated mitochondriopathy
(7) directly contributes to ROS generation, as has been observed in isolated endothelial cells (10). ROS and oxylipid metabolites are critical for the microbiocidal activity of phagocytes, wound healing, and the pathobiology of several inflammatory diseases including atherosclerosis. Since ROS can induce DNA damage and both are increased in diabetes $(11,12)$, the accumulation of both diabetes-associated mitochondrial and genomic DNA alterations are considered to be the consequence of abnormal mitochondrial ROS production. Multiple cellular enzymes generate ROS signatures in distinct subcellular venues (13), primarily superoxide (e.g., NOX1, NOX2, mitochondrial complexes), with rapid dismutation to $\mathrm{H}_{2} \mathrm{O}_{2}$ or direct $\mathrm{H}_{2} \mathrm{O}_{2}$ elaboration (e.g., NOX4, acyl-CoA oxidase, xanthine oxidase). These local intracellular ROS signatures, which are dynamic and elicited in response to intracellular and extracellular cues, are highly regulated and critical second messengers in metabolism and signal transduction (14). The interrelationships between distinct intracellular ROS metabolism and extracellular oxidative stress are poorly understood but are of emerging importance in the pathogenesis of organ dysfunction associated with aging and chronic diseases including diabetes (14).

In this issue of the JCI, Dugan and colleagues examine the role of mitochondrial-derived superoxide in diabetic kidney disease (15). Implementing multiple validated and unconventional methods of superoxide assessment, they demon- 